\title{
A NEW APPROACH TO THE CRITICAL VALUE THEORY
}

\author{
W. MAYER
}

The well known inequalities of M. Morse have as algebraical foundation the rank-equations

$$
\begin{array}{r}
r B_{i}\left(\Sigma-\Sigma_{1}\right)=r B_{i}(\Sigma)-r B_{i}\left(\Sigma_{1}\right)+r D_{i}\left(\Sigma_{1}, \Sigma\right)+r D_{i-1}\left(\Sigma_{1}, \Sigma\right) \\
i=0,1,2, \cdots .
\end{array}
$$

These formulas hold for any topological group-system $\Sigma$, any subsystem $\Sigma_{1}$ of $\Sigma$, and the difference-system $\Sigma-\Sigma_{1}$. (W. Mayer, Topologische Gruppensysteme, Monatshefte für Mathematik und Physik, vol. 47 (1938); henceforth referred to as M, TG.) Here $B_{i}(\Sigma)$ denotes the $i$-dimensional Betti group of $\Sigma$, while $B_{i}\left(\Sigma_{1}\right)$ and $B_{i}\left(\Sigma-\Sigma_{1}\right)$ are these groups for $\Sigma_{1}$ and $\Sigma-\Sigma_{1}$ respectively. The symbol $r()$, of course, stands for the rank of the group in the parentheses. By $D_{i}\left(\Sigma_{1}, \Sigma\right)$ we mean the subgroup of $B_{i}\left(\Sigma_{1}\right)$ containing all the classes of this group whose elements bound in $\Sigma$.

The formula (I) was first derived for the case of a complex in Lefschetz' Topology, 1930 (p. 150), and independently for the complex modulo 2 by J. Rybarz, Monatshefte für Mathematik und Physik (1931).

In the generality needed here the proof of (I) is given in M, TG (pp. 54-57), under the assumption, of course, that all the ranks appearing in (I) are finite, since otherwise the formula would be meaningless. But the proof there given shows also that

(a) $r B_{i}\left(\Sigma-\Sigma_{1}\right)=\infty$ implies that either $r B_{i}(\Sigma)$ or $r D_{i-1}\left(\Sigma_{1}, \Sigma\right)$, or both, are infinite;

(b) $r B_{i}\left(\Sigma-\Sigma_{1}\right)$ finite implies $r D_{i-1}\left(\Sigma_{1}, \Sigma\right)$ finite, and if in addition $r B_{i}\left(\Sigma_{1}\right)$ is finite then $r B_{i}(\Sigma)$ is finite too; and

(c) $r B_{i}\left(\Sigma-\Sigma_{1}\right)=0$ implies $r D_{i-1}\left(\Sigma_{1}, \Sigma\right)=0$ and if in addition $r B_{i}\left(\Sigma_{1}\right)$ is finite, then $r B_{i}\left(\Sigma_{1}\right)=r B_{i}(\Sigma)+r D_{i}\left(\Sigma_{1}, \Sigma\right)$.

As an immediate consequence of equations (I) we notice the inequality

$$
r B_{i}(\Sigma) \leqq r B_{i}\left(\Sigma_{1}\right)+r B_{i}\left(\Sigma-\Sigma_{1}\right),
$$

which is true whenever the terms on the right are finite (remark (b)) and trivial otherwise. The next step in attaining the Morse inequalities is the application of (I) to $m+2$ topological group-systems satisfying the inclusion relations

$$
\Sigma_{m} \supset \Sigma_{m-1} \supset \cdots \supset \Sigma_{0} \supset \Sigma_{-1}
$$


with $\Sigma_{-1}$ empty (all $L_{i}\left(\Sigma_{-1}\right)$ being zero-groups). Assuming that all ranks involved are finite, we get by summation on $k$ in

$$
\begin{aligned}
r B_{i}\left(\Sigma_{k}-\Sigma_{k-1}\right)= & r B_{i}\left(\Sigma_{k}\right)-r B_{i}\left(\Sigma_{k-1}\right)+r D_{i}\left(\Sigma_{k-1}, \Sigma_{k}\right) \\
& +r D_{i-1}\left(\Sigma_{k-1}, \Sigma_{k}\right)
\end{aligned}
$$

the formula

$$
\begin{aligned}
\sum_{k=0}^{m} r B_{i}\left(\Sigma_{k}\right. & \left.-\Sigma_{k-1}\right) \\
& =r B_{i}\left(\Sigma_{m}\right)+\sum_{k=1}^{m} r D_{i}\left(\Sigma_{k-1}, \Sigma_{k}\right)+r D_{i-1}\left(\Sigma_{k-1}, \Sigma_{k}\right),
\end{aligned}
$$

since all the $r B_{i}\left(\Sigma_{-1}\right), r D_{i}\left(\Sigma_{-1}, \Sigma_{0}\right)$ are zero, $\Sigma_{-1}$ being empty.

Let $\Sigma_{m}$ be a neighborhood-space and let $\Sigma_{k}, k=m-1, \cdots,-1$, be subspaces of $\Sigma_{m}$ satisfying the inclusion relation (1). With the introduction of singular simplices and chains modulo 2 each space $\Sigma_{k}$ gives rise to a topological group system, which we also denote by $\Sigma_{k}$. (The $i$-dimensional complexes $K^{i}$ are the finite chains of singular simplices.) The relations (1) are then inclusion relations for the so-constructed group-systems $\Sigma_{k}$, and for these systems relation (3) will hold provided the ranks involved are finite. The finiteness of all these ranks will follow from the finiteness of those of the left side of (3), that is, of the

$$
r B_{i}\left(\Sigma_{k}-\Sigma_{k-1}\right)
$$

If these are finite, we see from (2) that $r B_{i}\left(\Sigma_{0}\right)\left(=r B_{i}\left(\Sigma_{0}-\Sigma_{1}\right)\right)$ is finite, and thus from (2) and remark (b) we conclude that $r B_{i}\left(\Sigma_{1}\right)$ is finite. So, step-by-step, using (2) and remark (b) we find that all the ranks $r B_{i}\left(\Sigma_{k}\right)$ are finite. Since $D_{i}\left(\Sigma_{k-1}, \Sigma_{k}\right) \subset B_{i}\left(\Sigma_{k-1}\right)$, the ranks of the groups $D_{i}$ are finite too. We have then the result:

The equations (3) hold for the group-systems $\Sigma_{k}$ if only the ranks appearing on the left sides of these equations are finite.

It is an interesting fact, in view of its geometrical implications, that the finiteness of the ranks $r B_{i}\left(\Sigma_{k}-\Sigma_{k-1}\right)$ has as a consequence the finiteness of the Betti numbers $r B_{i}\left(\Sigma_{k}\right)$. As an additional remark it may be noticed that equations (3) can be written in the form of Morse's inequalities (of the strong type) if (3) holds for all indices $i$. Denoting by $M_{i}$ the left sides of (3):

$$
M_{i}=\sum_{k=0}^{m} r B_{i}\left(\Sigma_{k}-\Sigma_{k-1}\right)
$$


and successively eliminating the sums with terms $r D_{i}$, we get

$$
\begin{gathered}
M_{0} \geqq R_{0}, \\
M_{1}-M_{0} \geqq R_{1}-R_{0} \\
M_{2}-M_{1}+M_{0} \geqq R_{2}-R_{1}+R_{0}, \\
\cdot \cdot \cdot \cdot \cdot \cdot \cdot \cdot \cdot \cdot \cdot \cdot \cdot \cdot \cdot \cdot, \\
M_{n}-M_{n-1}+\cdots \pm M_{0}=R_{n}-R_{n-1}+\cdots \pm R_{0},
\end{gathered}
$$

where $n$ is the dimension of the topological system $\Sigma_{m}$ and $R_{i}$ stands for $r B_{i}\left(\Sigma_{m}\right)$, the $i$ th Betti number of $\Sigma_{m}$. The inequalities $\left(3^{\prime}\right)$, however, are not fully equivalent to equations (3) since they result only if (3) holds for all the dimensions $i$ from zero up to $n$, the dimension of $\Sigma_{m}$. In addition, in (3) the difference

$$
\left(M_{i}-M_{i-1}+\cdots \pm M_{0}\right)-\left(R_{i}-R_{i-1}+\cdots \pm R_{0}\right)
$$

is shown to be $\sum r D_{i}\left(\Sigma_{k-1}, \Sigma_{k}\right)$, which fact, of course, is of importance since it readily yields the conditions for the equality sign in $\left(3^{\prime}\right)$.

A subdivision of the space $\Sigma_{m}$ by subspaces $\Sigma_{k}$ of $\Sigma_{m}$ can be obtained by the use of a real bounded function $\Im$ defined on $\Sigma_{m}$. Let $\sigma_{0}$ and $\sigma_{m}$ be the greatest lower and least upper bound of $\Im$ respectively, so that

$$
\sigma_{m} \geqq \Im \geqq \sigma_{0} ;
$$

then intermediate values $\sigma_{k}, k=1, \cdots, m-1$, and $\sigma_{-1}<\sigma_{0}$ may be introduced and subjected to the inequalities

$$
\sigma_{m}>\sigma_{m-1}>\cdots>\sigma_{1}>\sigma_{0}>\sigma_{-1}
$$

if $\Im$ is not constant on $\Sigma_{m}$. If $\Im$ is constant, (6) can be reduced to $\sigma_{0}>\sigma_{-1}$.

We now define the subspace $\Sigma_{k}$ as the set of all points $P$ of $\Sigma_{m}$ such that $\Im(P) \leqq \sigma_{k}$, that is, symbolically,

$$
\Sigma_{k} \equiv\left\{\Im \leqq \sigma_{k}\right\}
$$

(We observe that in this notation the subspace $\Sigma_{m}$ defined in (7) coincides with the space originally so denoted.)

Constructing the corresponding topological group-systems, denoted likewise by $\Sigma_{m}$ (with $\Sigma_{-1}$ consisting of zero groups only), we see that equations (3) hold if only the sum $M_{i}$ of (4) is finite. This shows the full generality of these equations, in which only the concepts of neighborhood space and its partition by subspaces (not necessarily defined by a function $\Im$ ) are used. (Later on the validity of formula 
(3) will be shown for a partition of the space by a countable set of subspaces.)

Let $\Sigma_{m}$ be a compact Riemannian manifold and $\Im$ a function of class $C^{2}$ defined on $\Sigma_{m}$ and having a finite number only of stationary points. Corresponding to these points there are a finite number of stationary values $\sigma_{k}, k=0,1, \cdots, m$, with $\sigma_{0}$ and $\sigma_{m}$ the absolute minimum and maximum respectively. Using these values in defining the $\Sigma_{k}$ as previously described and denoting by the same letters $\Sigma_{k}$ the corresponding group-systems, we again arrive at equations (3), given the finiteness of their left sides $M_{i}$.

Denote by $\Sigma_{k}^{*}$ the space of all points $P$ of $\Sigma_{m}$ with the property $\Im(P)<\sigma_{k}$; then obviously $\Sigma_{k} \subset \Sigma_{k}$.

By definition (Seifert-Threlfall, Variationsrechnung im Grossen, §4; we refer to this henceforth as S.T.) the type numbers $m_{i}(\sigma)$ for any value $\sigma$ of $\Im$ are the ranks of the Betti groups of $\Sigma_{(\sigma)}-\Sigma_{(\sigma)}$, where $\Sigma_{(\sigma)}$ and $\Sigma_{(\sigma)}$ are the point sets $\{\Im \leqq \sigma\}$ and $\{\Im<\sigma\}$ respectively. The value $\sigma$ is called critical if some of the $m_{i}(\sigma)$ are different from zero.

Only a stationary value (that is, a value belonging to a stationary point) can be critical, so that only for the stationary values $\sigma_{k}$, $k=0,1, \cdots, m$, are the type numbers

$$
m_{i}\left(\sigma_{k}\right)=r B_{i}\left(\Sigma_{h}-\Sigma_{k}\right)
$$

not all necessarily zero. In consequence of our assumption all the stationary points are isolated and thus their contribution to the corresponding $m_{i}\left(\sigma_{k}\right)$ will be finite (S.T., $\left.\$ 10\right)$. Since only a finite number of stationary points belong to a stationary value $\sigma_{k}, m_{i}\left(\sigma_{k}\right)$ will be finite (S.T., p. 87).

If therefore we can prove that

$$
r B_{i}\left(\Sigma_{k}-\Sigma_{k}\right)=r B_{i}\left(\Sigma_{k}-\Sigma_{k-1}\right),
$$

then not only is (3) verified, but its left side is shown to be the sum of all the type numbers of dimension $i$. Thus, in proving isomorphisms between the Betti groups of the systems

$$
\Sigma_{k}-\Sigma_{k}
$$

and

$$
\Sigma_{k}-\Sigma_{k-1}
$$

for $k=0,1, \cdots, m$, we likewise prove (9) and thereby (3).

The above isomorphisms are included in the following two statements:

(a) Each class of $B_{i}\left(\Sigma_{k}-\Sigma_{k-1}\right)$ lies in a definite class of $B_{i}\left(\Sigma_{k}-\Sigma_{k}^{*}\right)$. 
(b) Each class of $B_{i}\left(\Sigma_{k}-\Sigma_{k}\right)$ contains one and only one class of $B_{i}\left(\Sigma_{k}-\Sigma_{k-1}\right)$.

Indeed, (a) and (b) establish a correspondence between the classes of the above groups which is obviously an isomorphism.

As to the proof of the statements, (a) is almost self-evident, since an $i$-cycle of $\Sigma_{k}-\Sigma_{k-1}$ is an $i$-cycle of $\Sigma_{k}-\Sigma_{k}$, and an $i$-cycle of $\Sigma_{k}-\Sigma_{k-1}$ which is homologous to zero in $\Sigma_{k}-\Sigma_{k-1}$ is easily seen to be an $i$-cycle of $\Sigma_{k}-\Sigma_{k}$ homologous to zero in $\Sigma_{k}-\Sigma_{k}^{*}$.

We now prove the first part of (b), that is, each class of $B_{i}\left(\Sigma_{k}-\Sigma_{k}^{*}\right)$ contains a class of $B_{i}\left(\Sigma_{k}-\Sigma_{k-1}\right)$. This is by no means evident, and to prove it we must make use of $\Im$-deformations, which in the case we are considering will exist. Suppose given $\left\{Z^{i}\right\} \subset B_{i}\left(\Sigma_{k}-\Sigma_{k}\right)$; then there exists a $K^{i-1} \subset \Sigma_{k}$ such that

$$
R\left(Z^{i}\right)=K^{i-1} \text {. }
$$

But, on the compact point set $K^{i-1}$, the continuous function $\Im$ has somewhere a maximum which, of course, is smaller than $\sigma_{k}$, $\left(K^{i-1} \subset \Sigma_{k}\right)$. Thus $K^{i-1}$ lies in some $\Sigma^{*}$ defined by $\left\{\Im \leqq \sigma_{k}-\epsilon\right\}$, where $\epsilon>0$ may be so chosen that $\sigma_{k}-\epsilon>\sigma_{k-1}$. Then (S.T., p. 87) an $\Im$-deformation exists which carries the point set $\left\{\Im \leqq \sigma_{k}-\epsilon\right\}$ into the point set $\left\{\Im \leqq \sigma_{k-1}\right\}$. Thus there exist a $K^{i}$ on $\left\{\Im \leqq \sigma_{k}-\epsilon\right\}$ and a $K_{1}^{i-1}$ on $\left\{\Im \leqq \sigma_{k-1}\right\}$ such that

$$
R\left(K^{i}\right)=K^{i-1}+K_{1}^{i-1}
$$

Adding (10) to (11) we get

$$
R\left(Z^{i}+K^{i}\right)=K_{1}^{i-1}
$$

thus showing that $Z^{i}+K^{i}$, a cycle of the class $\left\{Z^{i}\right\}$ of $B_{i}\left(\Sigma_{k}-\Sigma_{k}\right)$, is a cycle of $\Sigma_{k}-\Sigma_{k-1}$. Hence $\left\{Z^{i}\right\}$ contains this cycle of $\Sigma_{k}-\Sigma_{k-1}$, and, according to (a), the class of $B_{i}\left(\Sigma_{k}-\Sigma_{k-1}\right)$ it represents.

We finally prove the second part of (b), namely: Each class of $B_{i}\left(\Sigma_{k}-\Sigma_{k}\right)$ contains only one class of $B_{i}\left(\Sigma_{k}-\Sigma_{k-1}\right)$. This again follows from the statement: If a cycle $Z^{i}$ of $\Sigma_{k}-\Sigma_{k-1}$ considered as a cycle of $\Sigma_{k}-\Sigma_{k}$ is homologous to zero in $\Sigma_{k}-\Sigma_{k}^{*}$, then this cycle will be homologous to zero in $\Sigma_{k}-\Sigma_{k-1}$. For this proof one must again make use of the $\Im$-deformation mentioned above. Let $Z^{i}$ be a cycle of $\Sigma_{k}-\Sigma_{k-1}$ homologous to zero in $\Sigma_{k}-\Sigma_{k}$; then there exist a $K^{i+1}$ in $\Sigma_{k}$ and a $K^{i}$ in $\Sigma_{k}$ such that

$$
R\left(K^{i+1}\right)=Z^{i}+K^{i},
$$

which shows that $Z^{i} \sim 0$ in $\Sigma_{k}-\Sigma_{k}$. Furthermore there will exist a $K^{i-1}$ in $\Sigma_{k-1}$ such that 


$$
R\left(Z^{i}\right)=K^{i-1},
$$

since $Z^{i}$ is a cycle of $\Sigma_{k}-\Sigma_{k-1}$. From (13) and (14) follows

$$
R\left(K^{i}\right)=K^{i-1} \text {. }
$$

The compact set $K^{i}$ of $\Sigma_{k}$, $\left(\Im<\sigma_{k}\right)$, will lie in some $\left\{\Im \leqq \sigma_{k}-\epsilon\right\}$. Using the $\Im$-deformation carrying $\left\{\Im \leqq \sigma_{k}-\epsilon\right\}$ into $\left\{\Im \leqq \sigma_{k-1}\right\}$, we establish the existence of a chain $K_{1}^{i+1}$ of $\left\{\Im \leqq \sigma_{k}-\epsilon\right\}$ and two chains $K_{1}^{i}$ and $L_{1}^{i}$ of $\left\{\Im \leqq \sigma_{k-1}\right\}$ satisfying the relation

$$
R\left(K_{1}^{i+1}\right)=K^{i}+K_{1}^{i}+L_{1}^{i}
$$

where $L_{1}^{i}$, as the deformation chain of $R\left(K^{i}\right)=K^{i-1}$ (which lies in $\Sigma_{k-1}$ ) will lie in $\Sigma_{k-1}$, by definition of an $\Im$-deformation. Adding the two relations (13) and (16), we get finally

$$
R\left(K^{i+1}+K_{1}^{i+1}\right)=Z^{i}+K_{1}^{i}+L_{1}^{i}
$$

where $K_{1}^{i}+L_{1}^{i} \subset \Sigma_{k-1}$ and $K^{i+1}+K_{1}^{i+1} \subset \Sigma_{k}$. This proves that $Z^{i} \sim 0$ in $\Sigma_{k}-\Sigma_{k-1}$.

We add that in proving (9) we made no use of the fact that either of the values $\sigma_{k}$ or $\sigma_{k-1}$ was stationary. We used only the existence of the above mentioned $\Im$-deformations, and these will always exist provided only that between $\sigma_{k}$ and $\sigma_{k-1}$ there are no stationary values. Given this condition, (9) always holds (of course, in the form $0=0$ when $\sigma_{k}$ is not a stationary value).

The theory so far developed is unable to deal with the concept of the "variational calculus in the large," since in this case the function $\Im$ defined on $\Sigma$ is unbounded above. We now adapt the theory to that case.

First we prove the following lemma:

Lemma. For three group-systems $\Sigma_{\alpha}, \alpha=1,2,3$, satisfying the inclusion relations

$$
\Sigma_{1} \subset \Sigma_{2} \subset \Sigma_{3}
$$

\section{the relation}

$$
r D_{i}\left(\Sigma_{1}, \Sigma_{3}\right) \leqq r D_{i}\left(\Sigma_{1}, \Sigma_{2}\right)+r D_{i}\left(\Sigma_{2}, \Sigma_{3}\right)
$$

holds.

Proof. Each class of $D_{i}\left(\Sigma_{1}, \Sigma_{3}\right)$ is contained in a definite class of $D_{i}\left(\Sigma_{2}, \Sigma_{3}\right)$ since each cycle of $\Sigma_{1}$ bounding in $\Sigma_{3}$ is a cycle of $\Sigma_{2}$ bounding in $\Sigma_{3}$ and each cycle of $\Sigma_{1}$ bounding in $\Sigma_{1}$ is a cycle of $\Sigma_{2}$ bounding in $\Sigma_{2}$. By correlating with an element of $D_{i}\left(\Sigma_{1}, \Sigma_{3}\right)$ that 
element of $D_{i}\left(\Sigma_{2}, \Sigma_{3}\right)$ which contains it, we define a homomorphism,

$$
D_{i}\left(\Sigma_{1}, \Sigma_{3}\right) \rightarrow D_{i}\left(\Sigma_{2}, \Sigma_{3}\right)
$$

whose kernel group is the subgroup of $D_{i}\left(\Sigma_{1}, \Sigma_{3}\right)$ consisting of all classes bounding in $\Sigma_{2}$, hence $D_{i}\left(\Sigma_{1}, \Sigma_{2}\right)$. Denoting the map-group of the homomorphism by $D_{i}^{\prime}\left(\Sigma_{2}, \Sigma_{3}\right)$ we have the isomorphism

$$
D_{i}\left(\Sigma_{1}, \Sigma_{3}\right)-D_{i}\left(\Sigma_{1}, \Sigma_{2}\right) \approx D_{i}^{\prime}\left(\Sigma_{2}, \Sigma_{3}\right)
$$

and the rank-equation

$$
r D_{i}\left(\Sigma_{1}, \Sigma_{3}\right)=r D_{i}\left(\Sigma_{1}, \Sigma_{2}\right)+r D_{i}^{\prime}\left(\Sigma_{2}, \Sigma_{3}\right),
$$

whence (19) follows (since $D_{i}^{\prime}\left(\Sigma_{2}, \Sigma_{3}\right) \subset D_{i}\left(\Sigma_{2}, \Sigma_{3}\right)$ ).

Combining (I) and (19) we derive

$$
r B_{i}\left(\Sigma_{3}-\Sigma_{1}\right) \leqq r B_{i}\left(\Sigma_{3}-\Sigma_{2}\right)+r B_{i}\left(\Sigma_{2}-\Sigma_{1}\right),
$$

thus showing that in subdividing a given partition we never decrease the sum $M_{i}$.

As for the validity of the proof given for (23), we have to establish the legality of the use of formula (I). This use will indeed be legitimate if the rank equations (I) hold for $B_{i}\left(\Sigma_{3}-\Sigma_{2}\right)$ and $B_{i}\left(\Sigma_{2}-\Sigma_{1}\right)$, since then all ranks in (I) for $\Sigma=\Sigma_{3}$ are finite, by remark (a) of page 838 and equation (19), and the employment of (I) is thus justified for all the three Betti groups appearing in (23). This condition will in fact be satisfied whenever (23) is used in the sequel.

Equation (23), however, is true without restrictions, as we show by the use of a formula of a more general type, namely, $\left(4^{\prime}\right)$ of page 46 in M, TG. By this formula, (18) implies the isomorphism $\Sigma_{3}-\Sigma_{2} \approx\left(\Sigma_{3}-\Sigma_{1}\right)-\left(\Sigma_{2}-\Sigma_{1}\right)$ which generalizes a well known groupisomorphism to group-systems. Using $\left(\mathrm{I}^{\prime}\right)$ for the replacement of $\Sigma$ and $\Sigma_{1}$ by $\Sigma_{3}-\Sigma_{1}$ and $\Sigma_{2}-\Sigma_{1}$ respectively we again arrive at formula (23), but now without any restriction.

After this remark we extend the proof of formula (3). Let the space $\Sigma$ be subdivided by a countable set of subspaces $\Sigma_{k}, k=-1,0,1, \cdots$, satisfying the inclusion relation

$$
\Sigma_{-1} \subset \Sigma_{0} \subset \Sigma_{1} \subset \cdots \subset \Sigma_{m} \subset \cdots \subset \Sigma
$$

with $\Sigma_{-1}$ empty and $\sum_{m=0}^{\infty}\left(\Sigma_{m}\right)=\Sigma$ (we write $\Sigma=\Sigma_{\infty}$ ). Then formula (3) holds for an infinite but bounded sum

$$
M_{i}=\sum_{k=1}^{\infty} r B_{i}\left(\Sigma_{k}-\Sigma_{k-1}\right)
$$


if only the partition satisfies an additional assumption on its topological nature, namely:

(C) Any compact point set of $\Sigma$ lies in some $\Sigma_{k}(k \neq \infty)$.

As a matter of fact, $(C)$ is satisfied if the partition (24) is defined by the level surfaces of a continuous real function $\Im$ unbounded above, the subspace $\Sigma_{k}$ being the set of all points $P$ such that $\Im(P) \leqq \sigma_{k}$ and $\sigma_{-1}<\sigma_{0}<\sigma_{1}<\ldots$ being an unbounded sequence, with $\sigma_{-1}<\Im(P)$ for all points $P$ of $\Sigma$.

Since by assumption $M_{i}$ is finite, the left side of (3) will be finite, and thus (3) holds for any $m$. Thus each sum

$$
\sum_{k=1}^{m} r D_{i}\left(\Sigma_{k-1}, \Sigma_{k}\right), \quad \sum_{k=1}^{m} r D_{i-1}\left(\Sigma_{k-1}, \Sigma_{k}\right)
$$

in (3), being not larger than $M_{i}$, converges as $m \rightarrow \infty$. As a further consequence, $r B_{i}\left(\Sigma_{m}\right)$ also converges as $m \rightarrow \infty$ :

$$
\lim _{m \rightarrow \infty} r B_{i}\left(\Sigma_{m}\right)=R_{i}^{\prime} .
$$

If only we can show that $R_{i}^{\prime}=r B_{i}(\Sigma)$, we shall have proved formula (3) for $m=\infty$, that is,

$$
M_{i}=R_{i}+\sum_{k=1}^{\infty} r D_{i}\left(\Sigma_{k-1}, \Sigma_{k}\right)+\sum_{k=1}^{\infty} r D_{i-1}\left(\Sigma_{k-1}, \Sigma_{k}\right),
$$

writing $R_{i}=r B_{i}(\Sigma)$.

Due to the convergence of the sequences (26) and (27), all of whose terms - as ranks - are positive integers, or zeros, there exists an integer $N$ such that for $k \geqq N$

$$
\begin{aligned}
& r B_{i}\left(\Sigma_{k}-\Sigma_{k-1}\right)=0, r D_{i}\left(\Sigma_{k-1}, \Sigma_{k}\right)=0, \\
& r D_{i-1}\left(\Sigma_{k-1}, \Sigma_{k}\right)=0, \quad r B_{i}\left(\Sigma_{k}\right)-R_{i}{ }^{\prime}=0 .
\end{aligned}
$$

Consequently, for $h>k>N$ the right-hand terms of the inequality

$$
r B_{i}\left(\Sigma_{h}-\Sigma_{k}\right) \leqq \sum_{j=k+1}^{h} r B_{i}\left(\Sigma_{j}-\Sigma_{j-1}\right)
$$

are zero, so that

$$
r B_{i}\left(\Sigma_{h}-\Sigma_{k}\right)=0 .
$$

From (31) and the assumption (C) we easily conclude that for $k>N$

$$
r B_{i}\left(\Sigma-\Sigma_{k}\right)=0 \text {. }
$$


In fact, (32) is proved if, for any $C^{i}$ representing an element of $B_{i}\left(\Sigma-\Sigma_{k}\right)$, we can show that $C^{i} \sim 0$ in $\Sigma-\Sigma_{k}$. As a compact set on $\Sigma$, ( $C^{i} \subset \Sigma$ with $R\left(C^{i}\right) \subset \Sigma_{k}$ ), $C^{i}$ lies in some $\Sigma_{h} \supset \Sigma_{k}$, and thus represents an element of $B_{i}\left(\Sigma_{h}-\Sigma_{k}\right)$. But since this group has the rank zero, by (31), $C^{i} \sim 0$ in $\Sigma_{h}-\Sigma_{k}$. Therefore there exists a $K^{i+1}$ and a $K^{i}$ satisfying

$$
K^{i+1} \subset \Sigma_{h}, \quad K^{i} \subset \Sigma_{k},
$$

such that

$$
R\left(K^{i+1}\right)=C^{i}+K^{i}
$$

this shows that $C^{i} \sim 0$ in $\Sigma-\Sigma_{k}$ and proves (32).

Using (32) in connection with the main formula (I) we have for $k>N$

$$
0=r B_{i}(\Sigma)-r B_{i}\left(\Sigma_{k}\right)+r D_{i}\left(\Sigma_{k}, \Sigma\right)+r D_{i-1}\left(\Sigma_{k}, \Sigma\right) .
$$

Remark. The use of (I) is again justified, since all ranks involved are finite. (For $r B_{i}(\Sigma)$ and $r D_{i-1}\left(\Sigma_{k}, \Sigma\right)$ see remark (b) on page 838; remember also that $r B_{i}\left(\Sigma_{k}\right)=R_{i}^{\prime}$ and $r D_{i}\left(\Sigma_{k}, \Sigma\right) \leqq r B_{i}\left(\Sigma_{k}\right)$.) According to our remark (c) on page 838 we have $r D_{i-1}\left(\Sigma_{k}, \Sigma\right)=0$ in formula (35). We shall prove that in addition $r D_{i}\left(\Sigma_{k}, \Sigma\right)=0$.

This again is shown if for any $C^{i}$ representing an element of $D_{i}\left(\Sigma_{k}, \Sigma\right)$ we can prove that $C^{i} \sim 0$ in $\Sigma_{k}$. Let $C^{i}$ be such an element; then there exists a $K^{i+1}$ such that

$$
C^{i}=R\left(K^{i+1}\right), \quad C^{i} \subset \Sigma_{k} .
$$

But, again, the compact set $K^{i+1}$ lies in some $\Sigma_{h} \supset \Sigma_{k}$, and thus $C^{i}$ represents an element of $D_{i}\left(\Sigma_{k}, \Sigma_{h}\right)$, whose rank is not larger than

$$
\sum_{j=k}^{h-1} r D_{i}\left(\Sigma_{j}, \Sigma_{j+1}\right)=0
$$

Therefore $r D_{i}\left(\Sigma_{k}, \Sigma_{h}\right)=0$, so that $C^{i}$ is homologous to zero in $\Sigma_{k}$, which was to be proved.

This shown, (35) reduces to

$$
r B_{i}\left(\Sigma_{k}\right)=r B_{i}(\Sigma), \quad k>N,
$$

thus proving (28).

If for the concept of variational calculus in the large $\left(f\left(x, x^{\prime}\right)\right.$ positive definite and of class $C^{2}$ ) we restrict ourselves to the case where only a finite number of stationary points (extremals) are below any $\Im$-level $\left(\Im=\int_{P}^{Q} f\left(x, x^{\prime}\right) d t>0\right)$ and if the sequence $\sigma_{k}, k=0,1, \cdots$, un- 
bounded above, includes all the stationary values, then $\Im$-deformations exist (S.T., p. 66), and can be used to prove homomorphisms between the systems $\left(\alpha_{k}\right)$ and $\left(\beta_{k}\right)$ on page 841 in exactly the same way as before.

Hence (9) will hold, and again the type numbers

$$
m_{i}\left(\sigma_{k}\right)=r B_{i}\left(\Sigma_{k}-\Sigma_{k}\right)
$$

are finite (S.T., $\$ 14$, Theorem II and $\$ 17$, Theorem II), so that (3) is true for any $m$.

In taking the sum $M_{i}=\Sigma m_{i}\left(\sigma_{k}\right)$, only critical values $\sigma_{k}$ (by their definition) add nonzero terms, and if noncritical values $\sigma$ were used in the construction, they can be omitted in writing $M_{i}$. Hence for this case too the Morse equations hold for a finite $M_{i}$.

Remark. If, in the case just considered, for any two subspaces $\Sigma^{\prime}$, $\Sigma^{\prime \prime}$ of $\Sigma$ the ranks of $D_{i}\left(\Sigma^{\prime}, \Sigma^{\prime \prime}\right)$ and $D_{i-1}\left(\Sigma^{\prime}, \Sigma^{\prime \prime}\right)$ are zero $\left(\Sigma^{\prime} \subset \Sigma^{\prime \prime}\right)$, then $M_{i}=R_{i}$. For finite $M_{i}$, of course, this follows from (28), but for infinite $M_{i}$ we have to prove the statement. First, from (I)-or more exactly from the proof leading to (I)-we have, for $\operatorname{any}^{1} k$

$$
r B_{i}(\Sigma) \geqq r B_{i}\left(\Sigma_{k}\right) \text {; }
$$

then for any $m$ we have

$$
\sum_{k=0}^{m} r B_{i}\left(\Sigma_{k}-\Sigma_{k-1}\right)=r B_{i}\left(\Sigma_{m}\right)
$$

Since the left side of (40) diverges for $m \rightarrow \infty$, so does $r B_{i}\left(\Sigma_{m}\right)$ diverge, and hence, by $(39), r B_{i}(\Sigma)$ cannot be finite.

Institute For Advanced Study

${ }^{1}$ In fact, we have $B_{i}\left(\Sigma-\Sigma_{k}\right) \approx B_{i}(\Sigma)-F_{i}\left(\Sigma, \Sigma_{k}\right), F_{i}\left(\Sigma, \Sigma_{k}\right) \approx B_{i}\left(\Sigma_{1}\right)$ (M, TG, pp. 55-57). 